\title{
On The Function Rings of Pointfree Topology
}

\author{
BERNHARD BANASCHEWSKI \\ Department of Mathematics and Statistics, McMaster University, 1280 Main Street \\ West, Hamilton, Ontario L8S 4K1, Canada \\ e-mail : iscoe@math.mcmaster.ca
}

\begin{abstract}
The purpose of this note is to compare the rings of continuous functions, integer-valued or real-valued, in pointfree topology with those in classical topology. To this end, it first characterizes the Boolean frames (= complete Boolean algebras) whose function rings are isomorphic to a classical one and then employs this to exhibit a large class of frames for which the functions rings are not of this kind. An interesting feature of the considerations involved here is the use made of nonmeasurable cardinals. In addition, the integer-valued function rings for Boolean frames are described in terms of internal lattice-ordered ring properties.
\end{abstract}

As is familiar, pointfree topology - that is, the setting of frames - shares with classical topology the fact that each basic entity (spaces in one case, frames in the other) has associated with it the ring of its real-valued continuous functions, and this in such a way that the correspondence for frames extends that for spaces. To be precise, if $\mathcal{R} L$ is the ring associated with a frame $L$ and $\mathfrak{O} X$ the frame of open sets of a space $X$ then the classical function $\operatorname{ring} C(X)$ is naturally isomorphic to $\mathcal{R}(\mathfrak{O} X)$. It may be added here that the correspondence $X \mapsto \mathfrak{O} X$ effects a full dual embedding into the category of frames of the category of Tychonoff spaces - the natural context for considering the rings $C(X)$.

Now, given that there is a large supply of non-spatial frames, that is, frames not isomorphic to any $\mathfrak{O} X$, the correspondence $L \mapsto \mathcal{R} L$ is certainly a proper extension of the correspondence $X \mapsto C(X)$, via the intervening $X \mapsto \mathfrak{O} X$. That, however, does not a priori exclude the possibility that every $\mathcal{R} L$ might be isomorphic to some $C(X)$ but in fact this is not the case, and one of the purposes of this note is to describe a method of verifying this. There are other ways of doing this, as will be discussed later; the present approach is to provide first a characterization of the Boolean frames $L$ with $\mathcal{R} L$ isomorphic to some $C(X)$ and then to show that this excludes all non-atomic $L$ of nonmeasurable cardinal.

Actually, it turns out to be convenient to consider these matters first for the

Received April 15, 2005.

2000 Mathematics Subject Classification: 06F25, 54C30, 54H10.

Key words and phrases: ring of continuous functions in pointfree topology, Boolean frames and their $\sigma$-characters, nonmeasurable cardinals, order complete $f$-rings with singular unit. 
rings of integer-valued continuous functions; the real-valued case will then readily follow from the results established there by simple additional considerations.

To begin with, we briefly recall the definition of the function rings involved here; a detailed account of the real-valued case can be found in Banaschewski [1] and certain aspects of either case are considered in Banaschewski [2]. For general background concerning frames we refer to Johnstone [7] or Vickers [8]. The realvalued continuous functions on a frame $L$ are the frame homomorphisms $\alpha, \beta, \gamma, \cdots$ : $\mathcal{L}(\mathbf{R}) \rightarrow L$ where $\mathcal{L}(\mathbf{R})$ is the frame of reals given by the generators $(p, q)$ for $p, q \in \mathbf{Q}$ and the defining relations

(R1) $(p, q) \wedge(r, s)=(p \vee r, q \wedge s)$

(R2) $(p, q) \vee(r, s)=(p, s)$ whenever $p \leq r<q \leq s$

(R3) $(p, q)=\bigvee\{(r, s) \mid p<r<s<q\}$

(R4) $\bigvee\{(p, q) \mid p, q \in \mathbf{Q}\}=e$, the top(=unit).

Further, the $\mathcal{L}(\mathbf{R}) \rightarrow L$ form an $\ell$-ring $\mathcal{R} L$ over $\mathbf{Q}$ whose operations are induced by those of $\mathbf{Q}$ as follows:

For $\diamond=+, \cdot, \wedge, \vee$,

$$
\alpha \diamond \beta(p, q)=\bigvee\{\alpha(r, s) \wedge \beta(t, u) \mid\langle r, s\rangle \diamond\langle t, u\rangle \subseteq\langle p, q\rangle\}
$$

where $\langle\cdot, \cdot\rangle$ stands for open interval in $\mathbf{Q}$ and the inclusion on the right means that $x \diamond y \in\langle p, q\rangle$ whenever $x \in\langle r, s\rangle$ and $y \in\langle t, u\rangle$.

For,$-(-\alpha)(p, q)=\alpha(-q,-p)$.

For any $r \in \mathbf{Q}$,

$$
\mathbf{r}(p, q)= \begin{cases}e & \text { if } p<r<q \\ 0 & \text { otherwise. }\end{cases}
$$

$\mathcal{R} L$ satisfies all $\ell$-ring identities which hold in $\mathbf{Q}$ and is therefore a commutative $f$-ring with unit $\mathbf{1}$ over $\mathbf{Q}$. Moreover, it is archimedean.

On the other hand, the $\ell$-ring $\mathfrak{Z} L$ of integer-valued continuous functions on $L$ has as its elements the maps $\alpha, \beta, \gamma, \cdots: \mathbf{Z} \rightarrow L$ such that

$$
\alpha(k) \wedge \alpha(\ell)=0 \text { for } k \neq \ell \text { and } \bigvee\{\alpha(m) \mid m \in \mathbf{Z}\}=e,
$$

while its operations are induced by those of $\mathbf{Z}$ as follows:

For $\diamond=+, \cdot, \wedge, \vee$,

$$
\begin{aligned}
\alpha \diamond \beta(m) & =\bigvee\{\alpha(k) \wedge \beta(\ell) \mid k \diamond \ell=m\} \\
(-\alpha)(m) & =\alpha(-m)
\end{aligned}
$$

$\mathbf{0}(m)=e$ for $m=0, \mathbf{1}(m)=e$ for $m=1$ (which forces the remaining $\mathbf{0}(m)$ and $\mathbf{1}(m)$ to be 0$)$.

$\mathfrak{Z} L$ satisfies all $\ell$-ring identical implications which hold in $\mathbf{Z}$, making it a commutative $f$-ring with unit $\mathbf{1}$ such that

$$
\alpha \wedge(\mathbf{1}-\alpha) \leq 0 \text { for all } \alpha \in \mathfrak{Z} L .
$$


We call the latter the $\mathbf{Z}$-identity. As an important consequence of this we note that the idempotents of $\mathfrak{Z} L$ are exactly the $\sigma \in \mathfrak{Z} L$ such that $\mathbf{0} \leq \sigma \leq \mathbf{1}$. Further, similar to $\mathcal{R} L, \mathfrak{Z} L$ is archimedean.

As already indicated earlier, the $\mathcal{R} L$ are related to the classical $C(X)$ by an isomorphism $C(X) \rightarrow \mathcal{R}(\mathfrak{O} X)$ for each space $X$, explicitly given by the correspondence

$$
a \mapsto \tilde{a}, \tilde{a}(p, q)=a^{-1}[\{\lambda \in \mathbb{R} \mid p<\lambda<q\}] .
$$

Similarly, there is an isomorphism $C(X, \mathbf{Z}) \rightarrow \mathfrak{Z}(\mathfrak{O} X)$ for each space $X$, taking $a \in C(X, \mathbf{Z})$ to $\hat{a} \in \mathfrak{Z}(\mathfrak{O} X)$ for which $\hat{a}(m)=a^{-1}[\{m\}]$.

Finally, a function ring $\mathcal{R} L$ or $\mathfrak{Z} L$ will be called classical if it is isomorphic to some $C(X)$ or $C(X, \mathbf{Z})$, respectively.

In the following, we shall restrict our considerations to Boolean frames, that is, complete Boolean algebras. Note that these are the most convenient source of non-spatial frames: a Boolean frame is spatial if and only if it is atomic. Also, recall that a map between frames is called a $\sigma$-frame homomorphism if it preserves finitary meets and (at most) countable joins. In particular, for any Boolean frame $L$, the $\sigma$-frame homomorphisms $L \rightarrow \mathbf{2}$ will be called the $\sigma$-characters of $L$. Further, $\Phi L$ will be the $\sigma$-frame spectrum of $L$, that is, its space of $\sigma$-characters, with basic open sets

$$
\Phi_{a}=\{\tau \in \Phi L \mid \tau(a)=1\} .
$$

It is clear that $a \mapsto \Phi_{a}$ is then a $\sigma$-frame homomorphism $L \rightarrow \mathfrak{O} \Phi L$, and $L$ will be said to have enough $\sigma$-characters whenever $\Phi_{a}=\emptyset$ implies $a=0$; note the latter holds if and only if the map $a \mapsto \Phi_{a}$ is a $\sigma$-frame embedding.

The basic result in this context is now the following.

Lemma 1. For any Boolean frame $L$, the $\ell$-ring homomorphisms $\xi: \mathfrak{Z} L \rightarrow \mathbf{Z}$ correspond exactly to the $\sigma$-characters $\tau: L \rightarrow \mathbf{2}$.

Proof. We first establish that (i) each $\xi: \mathfrak{Z} L \rightarrow \mathbf{Z}$ determines a $\tau: L \rightarrow \mathbf{2}$ and (ii) conversely, and then show that (iii) the resulting correspondences are inverse to each other.

(i) For any $a \in L$, let $\gamma_{a} \in \mathfrak{Z} L$ be defined by

$$
\gamma_{a}(1)=a, \gamma_{a}(0)=a^{*}, \gamma_{a}(m)=0 \text { for all } m \neq 0,1,
$$

where $a^{*}$ is the pseudocomplement of $a$ (which is actually the complement here since $L$ is Boolean). Note that these $\gamma_{a}$ are the analogues of the characteristic functions of open-closed sets of a topological space; accordingly, we refer to $\gamma_{a}$ as the characteristic function of $a$. As is familiar, $\gamma_{a}$ is an idempotent in $\mathfrak{Z} L$, and any idempotent $\sigma \in \mathfrak{Z} L$ is of this kind - in fact $\sigma=\gamma_{\sigma(1)}$. Now, for the given $\xi: \mathfrak{Z} L \rightarrow \mathbf{Z}$, consider the map $\tau: L \rightarrow \mathbf{2}$ such that

$$
\tau(a)=\xi\left(\gamma_{a}\right)
$$


resulting from the fact that $\xi\left(\gamma_{a}\right)$ is an idempotent in $\mathbf{Z}$. We claim this is a $\sigma$-frame homomorphism.

Since $\tau$ is clearly a $0 \wedge e$-homomorphism, we only have to show it preserves countable joins, and for this it is enough to check that it takes any countable partition to a cover. Let $\left\{a_{n} \mid n=1,2, \cdots\right\}$ then be a partition of this kind and $\alpha \in \mathfrak{Z} L$ such that $\alpha(n)=a_{n}$ for $n=1,2, \cdots$ and $\alpha(m)=0$ otherwise. Then, for $\sigma_{k}=\gamma_{a_{k}}$,

$$
(\mathbf{n}-\alpha)^{+}=\sum_{k=1}^{n}(n-k) \sigma_{k}
$$

for each $n$ : with the usual notation for the restriction of continuous function on $L$ to some $\downarrow a, a \in L$, given by the homomorphism $L \rightarrow \downarrow a$ taking $s$ to $s \wedge a$, we have for any $\ell=1,2, \cdots$

$$
(\mathbf{n}-\alpha)^{+} \mid a_{\ell}=\left(\mathbf{n}\left|a_{\ell}-\alpha\right| a_{\ell}\right)^{+}=\left\{\begin{array}{ll}
(\mathbf{n}-\ell) \mid a_{\ell} & \text { if } \ell \leq n \\
\mathbf{0} \mid a_{\ell} & \text { if } \ell>n
\end{array},\right.
$$

while

$$
\left(\sum_{k=1}^{n}(n-k) \sigma_{k}\right) \mid a_{\ell}=\left\{\begin{array}{ll}
(\mathbf{n}-\ell) \mid a_{\ell} & \text { if } \ell \leq n \\
\mathbf{0} \mid a_{\ell} & \text { if } \ell>n
\end{array} .\right.
$$

It follows that $(*)$ holds since two continuous functions which have the same restriction to each member of some cover are equal.

Now, if $\tau\left(a_{n}\right)=0$ and hence $\xi\left(\sigma_{n}\right)=0$ for all $n$ then by $(*)$

$$
(n-\xi(\alpha))^{+}=0 \text { so that } n \leq \xi(\alpha)
$$

for any $n$, a contradiction since $\mathbf{Z}$ is archimedean. It follows that some $\tau\left(a_{n}\right)=1$, and this proves the claim.

(ii) Since the joins involved in the definition of $\mathfrak{Z} L$ are all countable, the correspondence $L \mapsto \mathfrak{Z} L$ is already functorial for $\sigma$-frame homomorphisms so that $\tau$ induces an $\ell$-ring homomorphism $\mathfrak{Z} L \rightarrow \mathfrak{Z} 2$ taking $\alpha \in \mathfrak{Z} L$ to the composite $\tau \alpha$. On the other hand, $\mathbf{2} \cong \mathfrak{D} \mathbf{1}$ for the one-point space $\mathbf{1}$ so that

$$
\mathfrak{Z} 2 \cong C(1, \mathbf{Z}) \cong \mathbf{Z} .
$$

Explicitly, the resulting isomorphism $\varrho: \mathbf{Z 2} \rightarrow \mathbf{Z}$ has the form

$$
\varrho(\alpha)=m \text { if and only if } \alpha(m)=1
$$

and the desired $\xi: \mathfrak{Z} L \rightarrow \mathbf{Z}$ is therefore given by

$$
\xi(\alpha)=m \text { if and only if } \tau(\alpha(m))=1 .
$$

(iii) Now let $\xi: \mathfrak{Z} L \rightarrow \mathbf{Z}$ be determined by a given $\tau: L \rightarrow \mathbf{2}$ as in (ii) so that

$$
\xi(\alpha)=\varrho(\tau \alpha)=m \text { if and only if } \tau(\alpha(m))=1 .
$$


In particular, for $\alpha=\gamma_{a}$,

$$
\xi\left(\gamma_{a}\right)=\varrho\left(\tau \gamma_{a}\right)=1 \quad \text { if and only if } \tau\left(\gamma_{a}(1)\right)=1 \text { if and only if } \tau(a)=1,
$$

showing that $\tau$ is the same as the $\sigma$-frame homomorphism $L \rightarrow \mathbf{2}$ derived from its associated $\xi: \mathfrak{Z} L \rightarrow \mathbf{Z}$.

In the opposite direction, given any $\xi: \mathfrak{Z} L \rightarrow \mathbf{Z}$ with the corresponding $\tau: L \rightarrow$ 2 such that $\tau(a)=\xi\left(\gamma_{a}\right)$, we have for $\alpha \in \mathfrak{Z} L$

$$
\xi\left(\gamma_{\alpha(m)}\right)=\tau(\alpha(m))=(\tau \alpha)(m)=1 \quad \text { if and only if } \varrho(\tau \alpha)=m .
$$

Further, by a routine calculation in $\mathfrak{Z} L, \gamma_{\alpha(m)}=(\mathbf{1}-|\alpha-\mathbf{m}|)^{+}$so that $\xi\left(\gamma_{\alpha(m)}\right)=1$ if and only if $\xi(\alpha)=m$. It follows that $\varrho(\tau \alpha)=\xi(\alpha)$, showing that $\mathfrak{Z} L \rightarrow \mathbf{Z}$ derived from $\tau$ is just the originally given $\xi$.

Remark 1. It can be shown that any ring homomorphism $\mathfrak{Z} L \rightarrow \mathbf{Z}$ is actually an $\ell$-ring homomorphism so that the above result, and the consequences derived from it below, could be adjusted accordingly, but since their present form is sufficient for the purpose at hand it seemed just as well to leave it at that. In a different vein, we note that the present result could also be obtained with the aid of suitable facts concerning the functor $\mathfrak{Z}$ from frames to archimedean $f$-rings which satisfy the Z-identity $a \wedge(1-a) \leq a$ and its left-adjoint (Banaschewski [2]) but it seemed instructive to present a selfcontained proof here.

Now we have the following characterization.

Proposition 1. For a Boolean frame $L, \mathfrak{Z} L$ is classical if and only if $L$ has enough $\sigma$-characters.

Proof. $(\Rightarrow)$ If $\varphi: \mathfrak{Z} L \rightarrow C(X, \mathbf{Z})$ is the given isomorphism then, for each $x \in X$, the map $L \rightarrow \mathbf{2}$ taking $a \in L$ to $\varphi\left(\gamma_{a}\right)(x)$ is a $\sigma$-frame homomorphism by the proof of Lemma 1. Further, if $a \neq 0$ then $\varphi\left(\gamma_{a}\right) \neq 0$ so that $\varphi\left(\gamma_{a}\right)(x)=1$ for some $x \in X$, which proves the claim.

$(\Leftarrow)$ The map $L \rightarrow \mathfrak{D} \Phi L$ taking $a$ to $\Phi_{a}$, being a $\sigma$-frame homomorphism, induces an $\ell$-ring homomorphism $\varphi: \mathfrak{Z} L \rightarrow \mathfrak{Z}(\mathfrak{O} \Phi L)$ and since $\mathfrak{Z}(\mathfrak{O} \Phi L) \cong C(\Phi L, \mathbf{Z})$ it will be sufficient to show this is an isomorphism. To see that it is one-one, consider any $\alpha \in \mathfrak{Z} L$ such that $\varphi(\alpha)=\mathbf{0}$. Then

$$
\Phi_{e}=\varphi(\alpha)(0)=\Phi_{\alpha(0)},
$$

hence $\alpha(0)=e$ by the present hypothesis and therefore $\alpha=\mathbf{0}$.

It remains to prove $\varphi$ is onto. For this, we first show that every open-closed $U \subseteq \Phi L$ is some $\Phi_{s}$. Let

$$
s=\bigvee\left\{a \in L \mid \Phi_{a} \subseteq U\right\} .
$$

Then $\Phi_{a} \subseteq \Phi_{s}$ whenever $\Phi_{a} \subseteq U$ and hence $U \subseteq \Phi_{s}$. On the other hand, if $\Phi_{b} \subseteq U^{*}$ ( ${ }^{*}$ for pseudocomplement) then $a \wedge b=0$ for all $\Phi_{a} \subseteq U$, hence $s \wedge b=0$ so that 
$\Phi_{s} \cap \Phi_{b}=\emptyset$ and therefore $\Phi_{s} \cap U^{*}=\emptyset$. This shows $\Phi_{s} \subseteq U^{* *}=U$, and in all then $U=\Phi_{s}$.

Now, for any $\beta \in \mathfrak{Z}(\mathfrak{O} \Phi L), \beta(m)=\Phi_{a_{m}}$ with a unique $a_{m} \in L$, as just shown, and putting $\alpha(m)=a_{m}$ then defines $\alpha \in \mathfrak{Z} L$ such that $\varphi(\alpha)=\beta$.

Remark 2. The appearance of $\sigma$-characters in the present context, which may at first seem oddly ad hoc and rather unmotivated, turns out to be quite natural and expected when viewed against the wider background. To see this, recall first the following details concerning zero-dimensional frames.

(i) A homomorphism $M \rightarrow L$ which induces an isomorphism $\mathfrak{Z} M \rightarrow \mathfrak{Z} L$ is called a $\mathfrak{Z}$-isomorphism.

(ii) $M$ is called $\mathfrak{Z}$-complete if any $\mathfrak{Z}$-isomorphism $N \rightarrow M$ is in fact an isomorphism.

(iii) Any $L$ has a $\mathfrak{Z}$-completion, meaning a $\mathfrak{Z}$-isomorphism $M \rightarrow L$ with $\mathfrak{Z}$ complete $M$.

(iv) For Boolean $L$, the $\mathfrak{Z}$-completion is given by $\mathfrak{H} L \rightarrow L$ where $\mathfrak{H} L$ is the frame of $\sigma$-ideals of $L$ and the map takes joins in $L$.

Now, one can show that, for any $\mathfrak{Z}$-complete $M, \mathfrak{Z} M$ is classical if and only if the spatial reflection map $M \rightarrow \mathfrak{O} \Sigma M$ ( $\Sigma$ for the usual frame spectrum) is a $\mathfrak{Z}$-isomorphism. Consequently $\mathfrak{Z} L$ is classical for any Boolean $L$ if and only if $\mathfrak{H} L \rightarrow \mathfrak{O} \Sigma \mathfrak{H} L$ is a $\mathfrak{Z}$-isomorphism, and here the $\sigma$-characters of $L$ naturally enter the picture: the frame homomorphism $\mathfrak{H} L \rightarrow 2$ correspond exactly to the $\sigma$-characters $L \rightarrow 2$ since the $\sigma$-frame embedding $L \rightarrow \mathfrak{H} L, a \mapsto \downarrow a=\{s \in L \mid s \leq a\}$ is the universal $\sigma$-frame homomorphism of $L$ to frames, providing an isomorphism $\Sigma \mathfrak{H} L \rightarrow \Phi L$. Note that this, then leads to a proof of $(\Rightarrow)$ of Proposition 1 without the need for Lemma 1.

Our next aim is to show that for a large class of Boolean frames $L, \mathfrak{Z} L$ can only be classical for trivial reason, that is, if $L$ itself is spatial. The key to this is the notion of nonmeasurable cardinal. Recall that a set $S$ is said to have nonmeasurable cardinal if, in our present language, the Boolean frame $\mathfrak{P} S$ has no $\sigma$-characters mapping every singleton to 0 . Turned around, this means every $\sigma$-character of $\mathfrak{P} S$ is in fact a frame homomorphism.

Now the desired result is as follows:

Proposition 2. Any Boolean frame $L$ of nonmeasurable cardinal with classical $\mathfrak{Z} L$ is spatial.

Proof. We refer to Gillman-Jerison [5] for the topological notions involved in the arguments below.

For any Boolean frame $L$ with enough $\sigma$-characters, the space $\Phi L$ is zerodimensional Hausdorff by definition and extremally disconnected because the Boolean algebra of its open-closed sets is $\left\{\Phi_{a} \mid a \in L\right\}$ by the proof of Proposition 1 and hence complete, being isomorphic to $L$. Further, any cozero set of $\Phi L$ is open-closed: for any such set $U, U=\bigcup U_{n}$ where $U_{n} \prec U$ (meaning $U \cup U_{n}^{*}=\Phi L$ ) 
which implies $U_{n} \subseteq U_{n}^{* *} \subseteq U$; now $U_{n}^{* *}$ is open-closed by extremal disconnectness so that $U_{n}^{* *}=\Phi_{a_{n}}$ for some $a_{n} \in L$, and therefore

$$
U=\bigcup \Phi_{a_{n}}=\Phi_{a} \text { for } a=\bigvee a_{n}
$$

In all, then, $\Phi L$ is an extremally disconnected $P$-space and as such discrete whenever it is of nonmeasurable cardinal $[5,12 \mathrm{H}])$. Furthermore, if $L$ is of nonmeasurable cardinal the same holds for $\Phi L$ so that it is discrete. Consequently, any singleton $\{\tau\}$ of $\Phi L$ is $\Phi_{s}$ for some $s \in L$, which is necessarily an atom in $L$, and this makes $L$ atomic, that is, spatial.

Remark 3. It is clear that this result easily leads to explicit descriptions of Boolean frames $L$ with non-classical $\mathfrak{Z} L$. As the simplest case, we note the countably generated atomless Boolean frames $L$ : countable generation makes $L$ the homomorphic image of a subframe of $\mathfrak{P}(\mathfrak{P N})$ which ensures nonmeasurable cardinal, and by the proposition atomlessness then trivially implies $\mathfrak{Z} L$ is non-classical. A convenient way to obtain Boolean frames of this kind is as follows. For any frame $L$, let $\mathfrak{B} L=\left\{a \in L \mid a=a^{* *}\right\}$ be its Booleanization, that is, the Boolean algebra of its regular elements. Then $\mathfrak{B} L$ is clearly countably generated whenever $L$ is. On the other hand, for regular $L$, the atoms of $\mathfrak{B} L$ are the same as the atoms of $L$ since regularity implies every element is a join of regular elements. Consequently, $\mathfrak{B} L$ is of the desired type for any countably generated atomless regular frame L. There are two distinct sources of such $L$, algebraic and topological. In the first case, take $L=\mathfrak{J} A$, the frame of ideals of a countable atomless Boolean algebra $A$ (such as the free Boolean algebra on a countable set), and in the second $L=\mathfrak{O} X$ for a second countable regular Hausdorff space $X$ without isolated points. As a final comment here it may be worth pointing out that this particular case is quite easily established directly, without recourse to the topological result used in the above general proof, the point being that any $\sigma$-frame homomorphism from a countably generated frame into a frame is in fact a frame homomorphism.

Remark 4. Obviously, Proposition 2 raises the question whether the existence oif measurable cardinals implies that of non-spatial Boolean frames with enough $\sigma$ characters. We note that while a measurable cardinal does produce a non-discrete extremally disconnected $P$-space, namely the Hewitt realcompactificaiton of the corresponding discrete space (Gillman-Jerison [5], 12H) this is not sufficient to provide the desired Boolean frames, and so far we have not been able to settle this matter.

We now move on to the case of real-valued functions. Their crucial connection with the earlier situation is given by the fact that, as in the classical setting, there is an $\ell$-ring embedding $\varrho_{L}: \mathfrak{Z} L \rightarrow \mathcal{R} L$ for any frame $L$ which, in this case, takes the form

$$
\varrho_{L}(\alpha)(p, q)=\bigvee\{\alpha(m) \mid p<m<q\}
$$

(Banaschewski [2]). 
As the first result here we have the following counterpart of Lemma 1.

Lemma 2. For any Boolean frame $L$, the $\ell$-ring homomorphisms $\xi: \mathcal{R} L \rightarrow \mathbf{R}$ correspond exactly to the $\sigma$-characters $\tau: L \rightarrow \mathbf{2}$.

Proof. For any $\xi: \mathcal{R} L \rightarrow \mathbf{R}$ as stated, the corresponding $\ell$-ring homomorphism $\xi \varrho_{L}: \mathfrak{Z} L \rightarrow \mathbf{R}$ maps $\mathfrak{Z} L$ into $\mathbf{Z}$ since its image is a $\mathbf{Z}$-ring and $\mathbf{Z}$ is the only subring of $\mathbf{R}$ of this kind. Consequently, by the proof of Lemma 1, we have a $\sigma$-frame homomorphism $\tau: L \rightarrow \mathbf{2}$ such that $\tau(a)=\xi \varrho_{L}\left(\gamma_{a}\right)$ where $\gamma_{a} \in \mathfrak{Z} L$, as before, is the characteristic function of $a \in L$. On the other hand, any $\sigma$-frame homomorphism $\tau: L \rightarrow \mathbf{2}$ induces an $\ell$-ring homomorphism $\mathcal{R} L \rightarrow \mathcal{R} \mathbf{2}$ because all the joins involved in the definition of $\mathcal{R} L$ are countable, and since $\mathcal{R} \mathbf{2} \cong C(\mathbf{1}) \cong \mathbf{R}$ this produces the desired $\xi: \mathcal{R} L \rightarrow \mathbf{R}$. Explicitly, by the well-known relation between $\mathbf{R}$ and $\mathcal{L}(\mathbf{R})$,

$$
\xi(\alpha)=\lambda \quad \text { if and only if } \tau(\alpha(p, q))=1 \text { whenever } p<\lambda<q
$$

for any $\alpha \in \mathcal{R} L$ and $\lambda \in \mathbf{R}$.

Next, if $\xi: \mathcal{R} L \rightarrow \mathbf{R}$ is determined in this way by a given $\tau: L \rightarrow \mathbf{2}$ then

$\xi \varrho_{L}\left(\gamma_{a}\right)=1$ if and only if $\tau\left(\bigvee\left\{\gamma_{a}(m) \mid p<m<q\right\}\right)=1$ whenever $p<1<q$,

where the join on the right is $a$ for any $p, q$ such that $0 \leq p<1<q \leq 2$. It follows that $\xi \varrho\left(\gamma_{a}\right)=1$ if and only if $\tau(a)=1$, showing that the $\sigma$-frame homomorphism $L \rightarrow \mathbf{2}$ derived from $\xi$ is the same as the original $\tau$.

Finally, given any $\xi: \mathcal{R} L \rightarrow \mathbf{R}$, let $\zeta: \mathcal{R} L \rightarrow \mathbf{R}$ be the $\ell$-ring homomorphism obtained from the $\sigma$-frame homomorphism $\tau: L \rightarrow \mathbf{2}$ determined by $\xi$ so that

$$
\zeta(\alpha)=\lambda \quad \text { if and only if } \xi \varrho_{L}\left(\gamma_{\alpha(p, q)}\right)=1 \text { whenever } p<\lambda<q .
$$

Now, for any $\alpha \geq \mathbf{0}$ in $\mathcal{R} L$,

$$
\alpha \wedge \mathbf{1} \leq \varrho_{L}\left(\gamma_{\operatorname{coz}(\alpha)}\right)
$$

where as usual

$$
\operatorname{coz}(\alpha)=\bigvee\{\alpha(p, 0) \vee \alpha(0, q) \mid p<0, q>0 \text { in } \mathbf{Q}\} ;
$$

trivially, $\alpha \wedge \mathbf{1}|\operatorname{coz}(\alpha) \leq \mathbf{1}| \operatorname{coz}(\alpha)$, and by the general properties of the cozero map coz:R $L \rightarrow L, \alpha \wedge \mathbf{1}\left|(\operatorname{coz}(\alpha))^{*}=\mathbf{0}\right| \operatorname{coz}(\alpha)^{*}$ so that $(*)$ holds on the cover $\left\{\operatorname{coz}(\alpha), \operatorname{coz}(\alpha)^{*}\right\}$ of $L$. It follows that $\xi(\alpha)>0$ implies $\xi \varrho_{L}\left(\gamma_{\operatorname{coz}(\alpha)}\right)=1$ since the latter is an idempotent in $\mathbf{R}$. Applying this to $\tilde{\alpha}(p, q)=(\alpha-\mathbf{p})^{+} \wedge(\mathbf{q}-\alpha)^{+}$and recalling that $\alpha(p, q)=\operatorname{coz}(\tilde{\alpha}(p, q))$ (Banaschewski [1]) we see that $\xi(\tilde{\alpha}(p, q))>0$ implies $\xi \varrho_{L}\left(\gamma_{\alpha(p, q)}\right)=1$. Now, if $\xi(\alpha)=\lambda$ then $\xi(\tilde{\alpha}(p, q))>0$ and hence $\xi \varrho_{L}\left(\gamma_{\alpha(p, q)}\right)=1$ whenever $p<\lambda<q$, which in turn implies $\zeta(\alpha)=\lambda$, showing that $\xi=\zeta$. 
Remark 5. As in the case of $\mathfrak{Z} L$ and $\mathbf{Z}$ noted in Remark 1, any ring homomorphism $\mathcal{R} L \rightarrow \mathbf{R}$ is in fact an $\ell$-ring homomorphism so that all our results involving these can also be adjusted accordingly.

As the real-valued analogue of Proposition 1 we now have.

Proposition 3. For a Boolean frame $L, \mathcal{R} L$ is classical if and only if $L$ has enough $\sigma$-characters.

Proof. $(\Rightarrow)$ Given the embedding $\varrho_{L}: \mathfrak{Z} L \rightarrow \mathcal{R} L$ it is immediate by our earlier arguments that $L$ has enough $\sigma$-characters if $\mathcal{R} L \cong C(X)$.

$(\Leftarrow)$ Recall from the proof of Proposition 1 that any cozero set of $\Phi L$ is openclosed under the present hypothesis.

Next, just as in the proof of Proposition 1, we have an embedding $\mathcal{R} L \rightarrow$ $\mathcal{R}(\mathfrak{O}(\Phi L))$ taking $\alpha \in \mathcal{R} L$ to $\check{\alpha} \in \mathcal{R}(\mathfrak{O}(\Phi L))$ such that $\check{\alpha}(p, q)=\Phi_{\alpha(p, q)}$, and it remains to show that the map $\alpha \mapsto \check{\alpha}$ is onto. Now, for any $\beta \in \mathcal{R}(\mathfrak{D}(\Phi L))$, $\beta(p, q)=\operatorname{coz}\left((\beta-\mathbf{p})^{+} \wedge(\mathbf{q}-\beta)^{+}\right)$and as just noted this is a $\Phi_{a}$. Hence there exists $\alpha \in \mathcal{R} L$ such that $\beta(p, q)=\Phi_{\alpha(p, q)}$ and therefore $\check{\alpha}=\beta$.

Further, by applying Propositions 3,1 , and 2 we immediately obtain the realvalued counterpart of Proposition 2.

Proposition 4. Any Boolean frame $L$ of nonmeasurable cardinal with classical $\mathcal{R} L$ is spatial.

Finally, it is obvious that Remarks 3 and 4, mutatis mutandis, apply to the present situation as well.

Remark 6. The following describes an alternative way of obtaining non-classical $\mathcal{R} L$, based on a number of topological facts. For general background we refer to Gillman-Jerison [5]; for the details specific to the present considerations see Hager [6]. Associated with any compact basically disconnected Tychonoff space $X$ one has the $\ell$-ring $D(X)$, given by the extended real-valued continuous functions on $X$ taking real values on some dense subset of $X$, which is known to be isomorphic to some $\mathcal{R} L$. Further, the $\ell$-ring homomorphisms $D(X) \rightarrow \mathbf{R}$ correspond to the $P$-points of $X$, and hence the $\mathcal{R} L$ involved will be non-classical whenever $X$ has no $P$-points. Finally, spaces $X$ of this kind are easily obtained: in fact, the projective cover of any second countable compact Hausdorff space without isolated points has this property. Naturally, one may wonder whether there is any connection between the present situation and that considered earlier. The following suggests this is indeed the case. It seems that, for any extremally disconnected compact Hausdorff space $X$, the present $D(X)$ is isomorphic to $\mathcal{R} L$ for the Boolean frame $L$ of regular open subsets of $X$, and that the $\sigma$-frame characters of the latter correspond exactly to the $P$-points of $X$. This would then make the examples of non-classical function rings just described also an application of Lemma 2. We leave the details to be settled some other time.

We close with a result which identifies the $\mathfrak{Z} L$ considered here within the general context of $\ell$-rings. For this we need the following notions. An $\ell$-ring is called 
order-complete if any non-void subset bounded above has a join (= least upper bound) and a $\mathbf{Z}$-ring is a commutative $f$-ring with unit which satisfies the $\mathbf{Z}$-identity: $a \wedge(1-a) \leq 0$ for all $a$. Next, an idempotent partition of an $\ell$-ring $A$ is a family $\left(u_{t}\right)_{t \in S}$ of idempotents with join equal to 1 such that $u_{t} \wedge u_{t^{\prime}}=0$ whenever $t \neq t^{\prime}$ in $S$. Further, we say that $A$ has effective idempotents if, for any idempotent partition $\left(u_{m}\right)_{m \in \mathbf{Z}}$ of $A$ indexed by $\mathbf{Z}$, there exists $a \in A$ such that $a u_{m}=m u_{m}$ for all $m \in \mathbf{Z}$. Finally, associated with each $\alpha \in \mathfrak{Z} L$ as before, we have its cozero element

$$
\operatorname{coz}(\alpha)=\bigvee\{\alpha(m) \mid 0 \neq m \in \mathbf{Z}\}
$$

and the corresponding map coz: $\mathfrak{Z} L \rightarrow L$ then satisfies the following for each $\alpha, \beta \in$ $3 L$ :

$\operatorname{coz}(\alpha)=0$ if and only if $\alpha=\mathbf{0}$

$\operatorname{coz}(\alpha \wedge \beta)=\operatorname{coz}(\alpha) \wedge \operatorname{coz}(\beta)$ whenever $\alpha, \beta \geq 0$

$\operatorname{coz}(\alpha) \leq \operatorname{coz}(\beta)$ if and only if $\alpha \leq \beta$ for idempotents $\alpha, \beta$.

Now the desired characterization is as follows.

Proposition 5. An $\ell$-ring is isomorphic to $\mathfrak{Z} L$ for some Boolean frame $L$ if and only if it is an order-complete $\mathbf{Z}$-ring with effective idempotents.

Proof. $(\Rightarrow)$ As shown in Banaschewski-Hong [3], $\mathfrak{Z} L$ is order-complete for a zerodimensional frame $L$ if and only if the Stone identity $a^{*} \vee a^{* *}=e$ holds in $L$, and since this is obviously the case for Boolean $L$ we have the desired completeness of $\mathfrak{Z} L$. Further, as already noted, $\mathfrak{Z} L$ is a $\mathbf{Z}$-ring, whatever the frame $L$. Finally, if $\left(\sigma_{m}\right)_{m \in \mathbf{Z}}$ is an idempotent partition of $\mathfrak{Z} L$ and $s \in L$ any upper bound of $\left\{\operatorname{coz}\left(\sigma_{m}\right) \mid m \in \mathbf{Z}\right\}$ then $\operatorname{coz}\left(\sigma_{m}\right) \leq s=\operatorname{coz}\left(\gamma_{s}\right)$ implies $\sigma_{m} \leq \gamma_{s}$ for all $m \in \mathbf{Z}$, by the rules for the coz-map listed above, and hence $\gamma_{s}=\mathbf{1}$, showing $s=e$. As a result, again in view of the above rules, we have $\alpha \in \mathfrak{Z} L$ such that $\alpha(m)=\operatorname{coz}\left(\sigma_{m}\right)$. Further, as already noted earlier, the idempotent $\tilde{\alpha}(m)=(\mathbf{1}-|\alpha-\mathbf{m}|)^{+}$is $\gamma_{\alpha(m)}$ and consequently the same as the given $\sigma_{m}$. Now, $\alpha \tilde{\alpha}(m)=m \tilde{\alpha}(m)$ by a simple calculation involving the $\mathbf{Z}$-identity: in any $\mathbf{Z}$-ring, if $b \geq 0$ then

$$
b \wedge\left((1-b)^{+}\right)=(b \wedge(1-b))^{+}=0,
$$

hence also $b(1-b)^{+}=0$, and the result follows by applying this to $|\alpha-\mathbf{m}|$.

$(\Leftarrow)$ For any order-complete $\mathbf{Z}$-ring $A$, the Boolean algebra $I d p A$ of its idempotents (consisting of all $u \in A$ such that $0 \leq u \leq 1$ by the $\mathbf{Z}$-identity) is complete and hence a Boolean frame. We shall show that $A \cong \mathfrak{Z}(\operatorname{Idp} A)$.

For any $a \in A$, let $\tilde{a}(m)=(1-|a-m|)^{+}$. Then, since binary meet in $\operatorname{Idp} A$ is the same as product,

$$
\tilde{a}(k) \wedge \tilde{a}(\ell)=\tilde{a}(k) \tilde{a}(\ell)=(\tilde{a}(\ell)-|a \tilde{a}(\ell)-k \tilde{a}(\ell)|)^{+}=(1-|\ell-k|)^{+} \tilde{a}(\ell)=0
$$

whenever $k \neq \ell$, where the third step follows by the calculation at the end of the above $(\Rightarrow)$ part. On the other hand, $\bigvee\{\tilde{a}(m) \mid m \in \mathbf{Z}\}=1$. In order to see this we 
shall show for the join $u$ involved here that

$$
(n-|a|)^{+} \wedge 1 \leq u \quad \text { for all } n .
$$

This will then imply $\left((n-|a|)^{+} v\right) \wedge v=0$ for $v=1-u$, hence $(n-|a|)^{+} v=$ $\left((n-|a|)^{+} v\right) v=0$ so that $n v \leq|a| v$ for all $n$, showing $v=0$ and therefore $u=1$ since $A$ is archimedean by order-completeness.

To obtain $(*)$, note first that

$$
|a| \wedge|a+1| \wedge|a-1| \wedge \cdots \wedge|a+n| \wedge|a-n| \leq(|a|-n)^{+},
$$

obtained by induction, using the identity $|a+k| \wedge|a-k| \leq|| a|-k|$ and the Z-identity. This then readily leads to

$$
\left(1-(|a|-n)^{+}\right)^{+} \leq \bigvee\left\{(1-|a-k|)^{+} \mid-n \leq k \leq n\right\} \leq u
$$

which proves $(*)$ since

$$
(1-((|a|-n) \vee 0)) \vee 0=((1+n-|a|) \wedge 1) \vee 0=(n+1-|a|)^{+} \wedge 1 .
$$

As a result, $(\tilde{a}(m))_{m \in \mathbf{Z}}$ is an idempotent partition of $A$, and the map $\tilde{a}: \mathbf{Z} \rightarrow I d p A$ taking $m$ to $\tilde{a}(m)$ belongs to $\mathfrak{Z}(\operatorname{Idp} A)$. Moreover, the resulting map $A \rightarrow \mathfrak{Z}(\operatorname{Idp} A)$ taking $a$ to $\tilde{a}$ is one-one and onto. Since $a \tilde{a}(m)=m \tilde{a}(m)$, as noted earlier, $\tilde{a}=\tilde{b}$ implies

$$
(|a-b| \wedge 1) \wedge \tilde{a}(m)=(|a-b| \wedge 1) \tilde{a}(m)=|a \tilde{a}(m)-b \tilde{b}(m)| \wedge \tilde{a}(m)=0
$$

for each $m$, and taking the join over all $m \in \mathbf{Z}$ then shows $|a-b| \wedge 1=0$ so that $a=b$. On the other hand, for any $\alpha \in \mathfrak{Z}(\operatorname{Idp} A)$ there exists $a \in A$ such that $a \alpha(m)=m \alpha(m)$ by hypothesis, and since

$$
\tilde{a}(m) \wedge \alpha(k)=(1-|k-m|)^{+} \alpha(k)=\left\{\begin{array}{cc}
\alpha(m) & \text { if } k=m \\
0 & \text { if } k \neq m
\end{array}\right.
$$

it follows that $\tilde{a}(m)=\alpha(m)$ for all $m \in \mathbf{Z}$, showing $\tilde{a}=\alpha$.

It remains to see that $a \mapsto \tilde{a}$ is an $\ell$-ring homomorphism. For any $\diamond=+, \cdot, \wedge, \vee$, $a, b \in A$, and $k, \ell, m \in \mathbf{Z}$ :

$$
(a \diamond b)^{\sim}(m) \tilde{a}(k) \tilde{b}(\ell)=(1-|(k \diamond \ell)-m|)^{+} \tilde{a}(k) \tilde{b}(\ell)=\left\{\begin{array}{cc}
\tilde{a}(k) \tilde{b}(\ell) & \text { if } k \diamond \ell=m \\
0 & \text { if } k \diamond \ell \neq m
\end{array}\right.
$$

the first step since multiplication by idempotents in $A$ preserves all $\ell$-ring operations, and taking the join over all $k, \ell \in \mathbf{Z}$ proves that

$$
(a \diamond b)^{\sim}(m)=\bigvee\{\tilde{a}(k) \wedge \tilde{b}(\ell) \mid k \diamond \ell=m\}=(\tilde{a} \diamond \tilde{b})(m)
$$

and hence $(a \diamond b)^{\sim}=\tilde{a} \diamond \tilde{b}$. The arguments for - and for the zero and the unit are analogous and left to the reader. 
Remark 7. There is an obvious alternative argument for $(\Leftarrow)$, based on the fact that the known representation of archimedean $f$-rings with unit in terms of the function rings $\mathcal{R} L$ provides an $\ell$-ring embedding $\zeta_{A}: A \rightarrow \mathfrak{Z}(\mathfrak{K} A)$ for any archimedean Z-ring $A$ where $\mathfrak{K} A$ is the frame of archimedean kernels of $A$, explicitly given by

$$
\zeta_{A}(a)(m)=[\tilde{a}(m)],
$$

[.] indicating principal $\ell$-ideal. In the end, though, it turns out this approach is hardly any shorter, and it therefore seemed just as well to provide a complete, selfcontained treatment here.

Remark 8. Given the restriction of our consideration to Boolean frames, that is, complete Boolean algebras, the $\ell$-rings $\mathfrak{Z} L$ considered here amount to a familiar algebraic construct which has been studied extensively: they are exactly the Boolean powers $\mathbf{Z}[L]$ of the $\ell$-ring $\mathbf{Z}$, as defined by Foster [4]. Consequently Proposition 5 may also be read as an internal characterization of the latter. In a similar vein, Proposition 2 says that, in the absence of measurable cardinals, any Boolean power of $\mathbf{Z}$ which is isomorphic to some $C(X, \mathbf{Z})$ is just an ordinary power $\mathbf{Z}^{S}$ of $\mathbf{Z}$.

Acknowledgment. Thanks are due to the Natural Sciences and Engineering Research Council of Canada for continuing support in the form of a discovery grant and to A. W. Hager for providing some most helpful background information.

\section{References}

[1] B. Banaschewski, The Real Numbers in Pointfree Topology. Textos de Matématica Sér. B, vol. 12, Departamento de Matématica da Universidade de Coimbra, 1997.

[2] B. Banaschewski, On the Function Ring Functor in Pointfree Topology, Appl. Categ. Structures, 13(2005), 305-328.

[3] B. Banaschewski and S. S. Hong, Completeness properties of function rings in pointfree topology, Comment. Math. Univ. Carolinae, 44(2003), 245-259.

[4] A. L. Foster, Generalized "Boolean" theory of universal algebras II: Identities and subdirect sums of functionally complete algebras, Math. Z., 59(1953), 191-199.

[5] L. Gillman and M. Jerison, Rings of Continuous Functions. Van Nostrand Reinhold Company, New York 1960.

[6] A. W. Hager, Algebras of measurable functions, Duke Math. J., 38(1971), 21-27.

[7] P. T. Johnstone, Stone spaces. Cambridge stud. adv. math., vol. 3, Cambridge University Press, Cambridge, 1982.

[8] S. Vickers, Topology via Logic. Cambridge Tracts Theoret. Comp. Sci., vol. 5, Cambridge University Press, Cambridge, 1985. 